\title{
Polymorphic Phase Transformation Pathways under Nanoconfinement:
}

\section{Flufenamic Acid}

Keke Zhang, ${ }^{\ddagger a, b}$ Noalle Fellah, ${ }^{\ddagger a}$ Vilmalí López-Mejías, ${ }^{\text {c,d }}$ Michael D. Ward*a

${ }^{a}$ Department of Chemistry and Molecular Design Institute, New York University, New York City, NY, 10003, USA; 'School of Chemical Engineering and Technology, State Key Laboratory of Chemical Engineering, Tianjin University, Tianjin 300072, People's Republic of China; 'Department of Chemistry, University of Puerto Rico, Río Piedras Campus, San Juan, Puerto Rico 00931, USA; 'Crystallization Design Institute, Molecular Sciences Research Center, University of Puerto Rico, San Juan, Puerto Rico 00926, USA.

† These authors contributed equally to this work.

\section{Supporting Information}

\section{TABLE OF CONTENTS}

\section{Methods and Materials}

Table S1. Crystallographic data for polymorphs of FFA forms I - VIII.

Figure S1. TGA data for FFA.

Figure S2. DSC data for FFA nanocrystals embedded within $100 \mathrm{~nm} \mathrm{CPG.}$

Figure S3. DSC data for FFA nanocrystals embedded within $140 \mathrm{~nm} \mathrm{CPG.}$

Figure S4. DSC data for FFA nanocrystals embedded within $200 \mathrm{~nm}$ CPG.

Figure S5. PXRD and DSC patterns of FFA mixed with nonporous glasses.

Figure S6. DSC endotherms for FFA form VIII.

Figure S7. PXRD patterns of FFA embedded in $50 \mathrm{~nm} \mathrm{CPG} \mathrm{pores} \mathrm{measured} \mathrm{at} \mathrm{room} \mathrm{temperature.}$

Figure S8. PXRD patterns of FFA embedded in $140 \mathrm{~nm}$ CPG pores measured at room temperature.

Figure S9. PXRD patterns of FFA embedded in $200 \mathrm{~nm} \mathrm{CPG} \mathrm{pores} \mathrm{measured} \mathrm{at} \mathrm{room} \mathrm{temperature.}$ 


\section{Methods and Materials}

Materials. Flufenamic acid (FFA, $\mathrm{C}_{14} \mathrm{H}_{10} \mathrm{~F}_{3} \mathrm{NO}_{2}, \geq 99 \%$ ) was purchased from Sigma-Aldrich (St. Louis, $\mathrm{MO}$, USA) and used without further purification. Controlled pore glass (CPG), a borate-silicate composite glass from which the borate phase is leached to produce a silica glass bead with a random pore network, was obtained from Biosearch Technologies (Petaluma, CA, USA) with nominal pore sizes of 30, 50, 100, 140 and $200 \mathrm{~nm}$. Porous glass with nominal pore sizes of 4 and $8 \mathrm{~nm}$, as well as nonporous glass beads, were purchased from Sigma-Aldrich (St. Louis, MO, USA). All the CPG beads were washed with boiling $70 \%$ nitric acid prior to use, then washed with deionized water and dried for $12 \mathrm{~h}$ under a vacuum. The acid-washed CPG was stored under air in a desiccator.

Confined crystal growth. CPG beads, a borate-silicate composite glass from which the borate phase has been leached to produce a silica glass bead with a random and continuous pore network, were mixed with FFA powder $(c a .2 .5 \mathrm{mg})$ in a hermetically sealed aluminum pan and differential scanning calorimetry (DSC) was used to infiltrate the CPG pores with FFA. The sample were heated from $25{ }^{\circ} \mathrm{C}$ to $150{ }^{\circ} \mathrm{C}$ at a heating rate of $10{ }^{\circ} \mathrm{C} / \mathrm{min}$, held at $150{ }^{\circ} \mathrm{C}$ for $5 \mathrm{~min}$. ensuring infiltration of molten FFA in the pores via capillary action. The samples were then cooled from $150{ }^{\circ} \mathrm{C}$ to $25{ }^{\circ} \mathrm{C}$ at a cooling rate of $10{ }^{\circ} \mathrm{C} / \mathrm{min}$ to induce FFA crystallization (supercooling). The mass ratio of FFA and CPG and pore volume provided by the manufacturer were used to calculate that $75 \%$ of the pores were filled. FFA crystallization on the surfaces of nonporous glass beads (NPG) was conducted using the same experimental procedure to compare the outcome of this crystallization to that when FFA polymorphs were produced under nanoconfinement. The molar enthalpy, $\Delta H$, was calculated from the DSC data by estimating the area under each endothermic peak (given in J) and dividing it by the total amount of FFA material embedded in the CPG matrices. Every experiment, including crystal melting and growth via DSC and characterization of polymorphic transitions, was repeated three times.

Characterization. Two-dimensional X-ray microdiffraction (2D $\mu$-XRD) was performed at room temperature with a Bruker D8 Discover General Area Diffraction System (GADDS) which was equipped

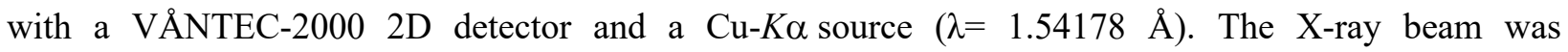
monochromated with a graphite crystal and collimated with a 0.5 capillary collimator (MONOCAP). The 
sample was loaded into a $0.8 \mathrm{~mm}$ Kapton capillary. Scans were performed in $20 \mathrm{~min}$. in room temperature with $\theta_{1}$ (incident angle of the X-ray beam) $=5^{\circ}$ and $\theta_{2}$ (detector angle) $=14^{\circ}$, respectively. One-dimensional (1D) diffraction patterns were integrated from the 2D XRD images over the entire range of azimuthal angles spanning $7^{\circ} \leq 2 \theta \leq 32^{\circ}$ range using the $\mathrm{XRD}{ }^{2} \mathrm{EVAL}$ program (version 2009.5-0; Bruker AXS Inc., Madison, WI, 2009). DSC was performed on a Pyris-1 (Perkin-Elmer Inc., Wellesley, MA) differential scanning calorimeter. An indium standard was used to calibrate the instrument and nitrogen was used as the purge gas. Thermal gravimetric analysis (TGA) data were collected on a Perkin-Elmer Diamond TG/DTA (Perkin-Elmer, Inc., Wellesley, MA), at a scan rate of $10{ }^{\circ} \mathrm{C} / \mathrm{min}$. The scan range was 25 to $155^{\circ} \mathrm{C}$ for TGA.

Table S1. Crystallographic data for polymorphs of FFA forms I - VIII. ${ }^{1}$

\begin{tabular}{|c|c|c|c|c|c|c|c|c|}
\hline & Form I & Form II & Form III & Form IV & Form V & Form VI & Form VII & Form VIII \\
\hline Space group & $\mathrm{P} 21 / \mathrm{c}$ & $\mathrm{P} 21 / \mathrm{c}$ & $\mathrm{C} 2 / \mathrm{c}$ & $\mathrm{P}-1$ & $\mathrm{P} 21 / \mathrm{c}$ & P-1 & $\mathrm{P} 21 / \mathrm{c}$ & $\mathrm{P}-1$ \\
\hline a $(\AA ̊)$ & $12.4157(12)$ & $10.8813(8)$ & $39.683(7)$ & $8.7589(2)$ & $26.6592(19)$ & $8.6485(2)$ & $14.9687(14)$ & $17.0047(14)$ \\
\hline b $(\AA)$ & $7.7528(7)$ & $10.2374(7)$ & $5.0556(8)$ & $11.6629(3)$ & $7.9007(2)$ & $11.5115(2)$ & $20.641(2)$ & $17.8870(14)$ \\
\hline c $(\AA ̊)$ & $12.6469(12)$ & $11.7487(10)$ & $11.963(2)$ & $20.0229(14)$ & $23.2430(6)$ & $38.895(3)$ & $7.9486(8)$ & $19.1941(15)$ \\
\hline$\alpha\left({ }^{\circ}\right)$ & 90 & 90 & 90 & $80.632(6)$ & 90 & $87.914(6)$ & 90 & $81.321(7)$ \\
\hline$\beta\left({ }^{\circ}\right)$ & $94.688(2)$ & $111.318(8)$ & $91.910(3)$ & $81.041(6)$ & $94.084(7)$ & $85.910(6)$ & $98.316(7)$ & $89.580(6)$ \\
\hline$\gamma\left({ }^{\circ}\right)$ & 90 & 90 & 90 & $73.532(5)$ & 90 & $72.260(5)$ & 90 & $78.560(7)$ \\
\hline $\begin{array}{c}\text { Cell volume } \\
\qquad\left(\AA^{3}\right)\end{array}$ & 1213.27 & 1219.21 & 2398.70 & 1922.33 & 4883.16 & 3678.32 & 2430.05 & 5655.09 \\
\hline $\mathbf{Z}, \mathbf{Z}^{\prime}$ & 4,1 & 4,1 & 8,1 & 6,3 & 16,4 & 12,6 & 8,2 & $19,9.5$ \\
\hline $\begin{array}{c}\text { Temperature } \\
\text { (K) }\end{array}$ & 90 & 95 & 90 & 273 & 95 & 85 & 85 & 85 \\
\hline Ref code & FPAMCA18 & FPAMCA17 & FPAMCA19 & FPAMCA15 & FPAMCA16 & FPAMCA14 & FPAMCA12 & FPAMCA13 \\
\hline
\end{tabular}




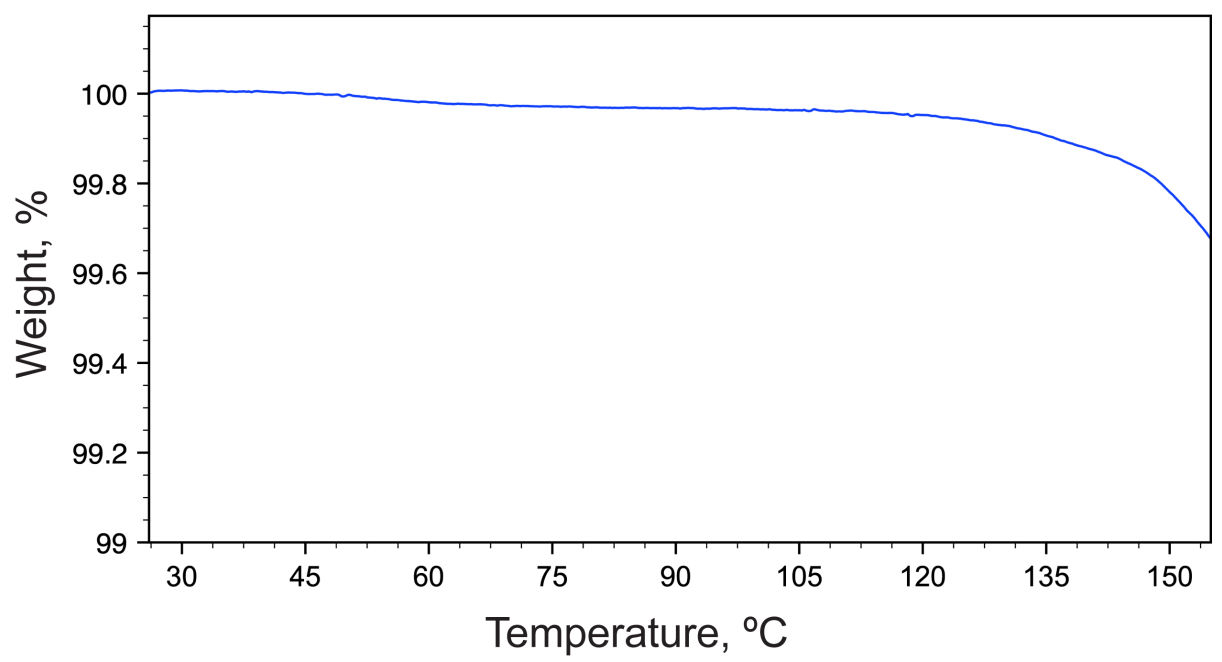

Figure S1. (A) Dynamic TGA thermograph of bulk form I. The sample was heated at a rate of $10^{\circ} \mathrm{C} / \mathrm{min}$ in the temperature range of 25 to $155^{\circ} \mathrm{C}$.

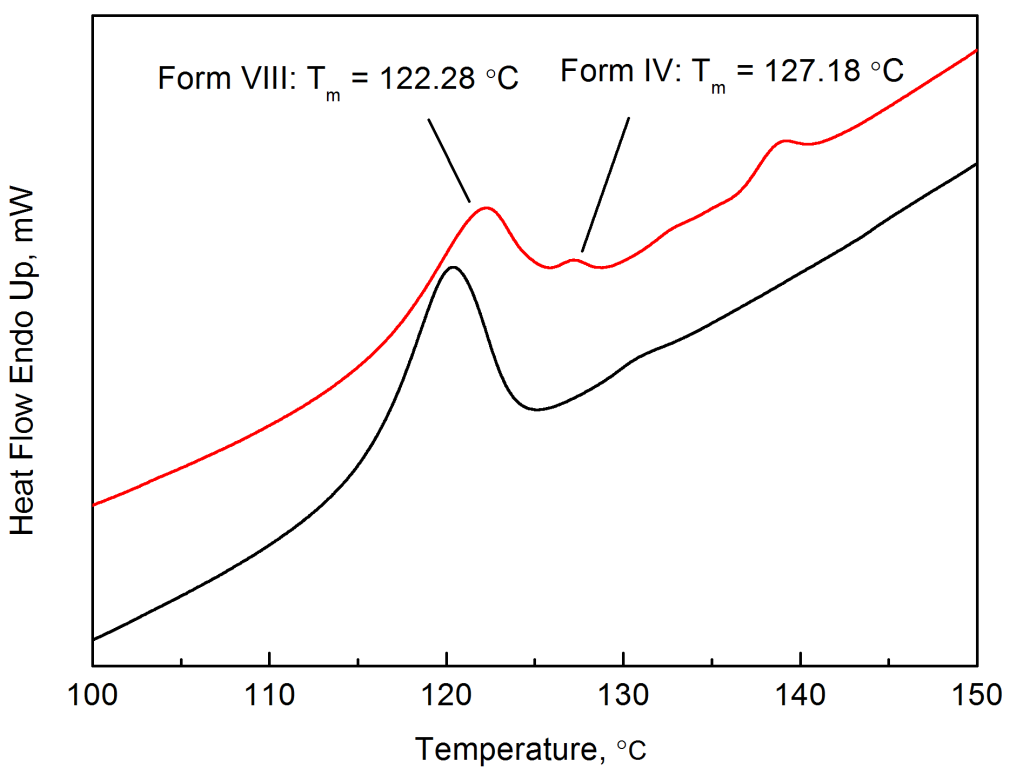

Figure S2. Representative DSC scans (uncorrected) for FFA nanocrystals embedded within $100 \mathrm{~nm}$ CPG: form VIII (initial scan, black) and form VIII after partial transformation to form IV (second scan, red). The same sample was used in both scans. The black trace corresponds to the DSC heating scan of form VIII nanocrystals embedded in $100 \mathrm{~nm}$ CPG, while red trace was obtained after melting the embedded form VIII nanocrystals, cooling to $25^{\circ} \mathrm{C}$ and holding at $25^{\circ} \mathrm{C}$ for $20 \mathrm{~min}$. The sample was heated and cooled at a rate of $10 \mathrm{~min} /{ }^{\circ} \mathrm{C}$ for both two scans in the temperature ranges 25 to $150{ }^{\circ} \mathrm{C}$. The second and third peaks in the red trace are attributed to the melting of form IV and a subsequently formed phase (likely form III), respectively. 


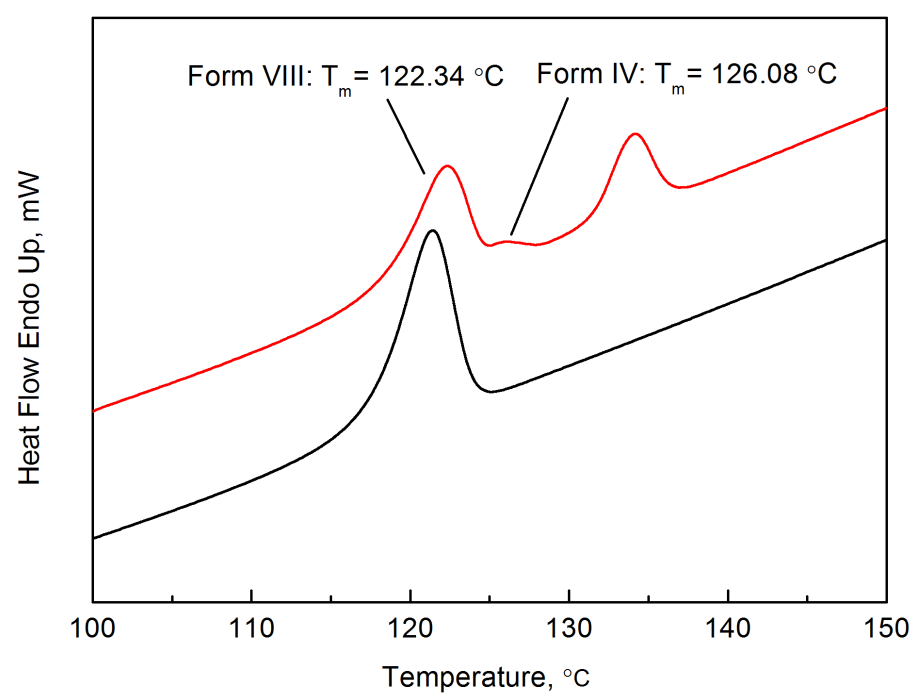

Figure S3. Representative DSC scans (uncorrected) for FFA nanocrystals embedded within $140 \mathrm{~nm}$ CPG: form VIII (initial scan, black) and form VIII after partial transformation to form IV (second scan, red). The same sample was used in both scans. The black trace corresponds to the initial DSC heating scan of form VIII nanocrystals embedded in 140 $\mathrm{nm}$ CPG, while the red trace was obtained after melting the embedded form VIII nanocrystals, cooling to $25{ }^{\circ} \mathrm{C}$ and holding at $25{ }^{\circ} \mathrm{C}$ for $20 \mathrm{~min}$. The sample was heated and cooled at a rate of $10 \mathrm{~min} /{ }^{\circ} \mathrm{C}$ for both two scans in the temperature ranges 25 to $150{ }^{\circ} \mathrm{C}$. The second and third peaks in the red trace are attributed to the melting of form IV and a subsequently formed phase (likely form III), respectively.

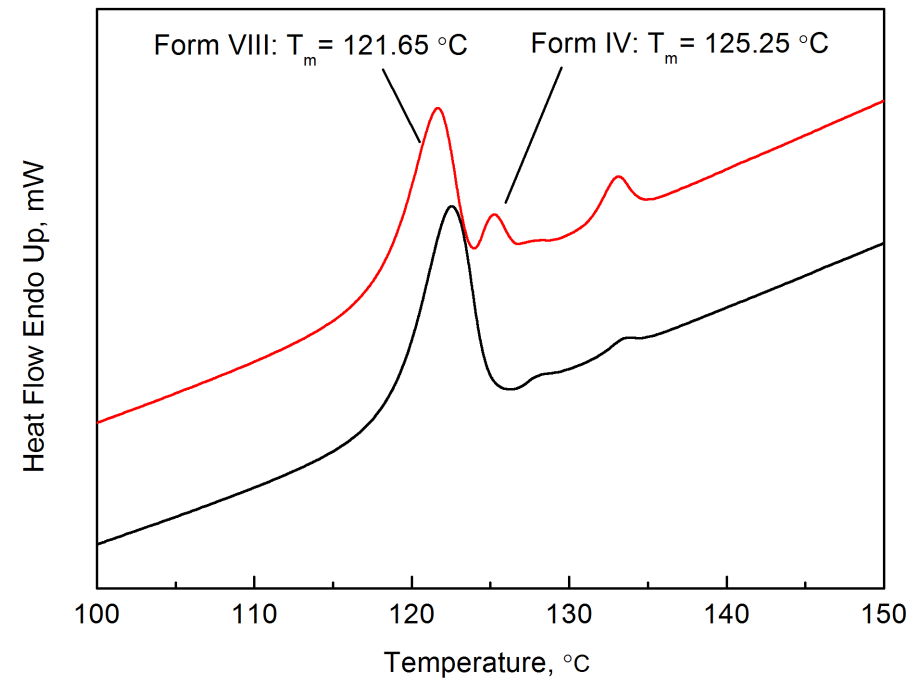

Figure S4. Representative DSC scans (uncorrected) for FFA nanocrystals embedded within $200 \mathrm{~nm}$ CPG: form VIII (initial scan, black) and form VIII after partial transformation to form IV (second scan, red). The same sample was used in both scans. The black trace corresponds to the DSC heating scan of form VIII nanocrystals embedded in $200 \mathrm{~nm}$ CPG, while red trace was obtained after melting the embedded form VIII nanocrystals, cooling to $25^{\circ} \mathrm{C}$ and holding at $25{ }^{\circ} \mathrm{C}$ for $20 \mathrm{~min}$. The sample was heated and cooled at a rate of $10 \mathrm{~min} /{ }^{\circ} \mathrm{C}$ for both two scans in the temperature ranges 25 to $150{ }^{\circ} \mathrm{C}$. The second and third peaks in the red trace are attributed to the melting of form IV and a subsequently formed phase (likely form III), respectively. 

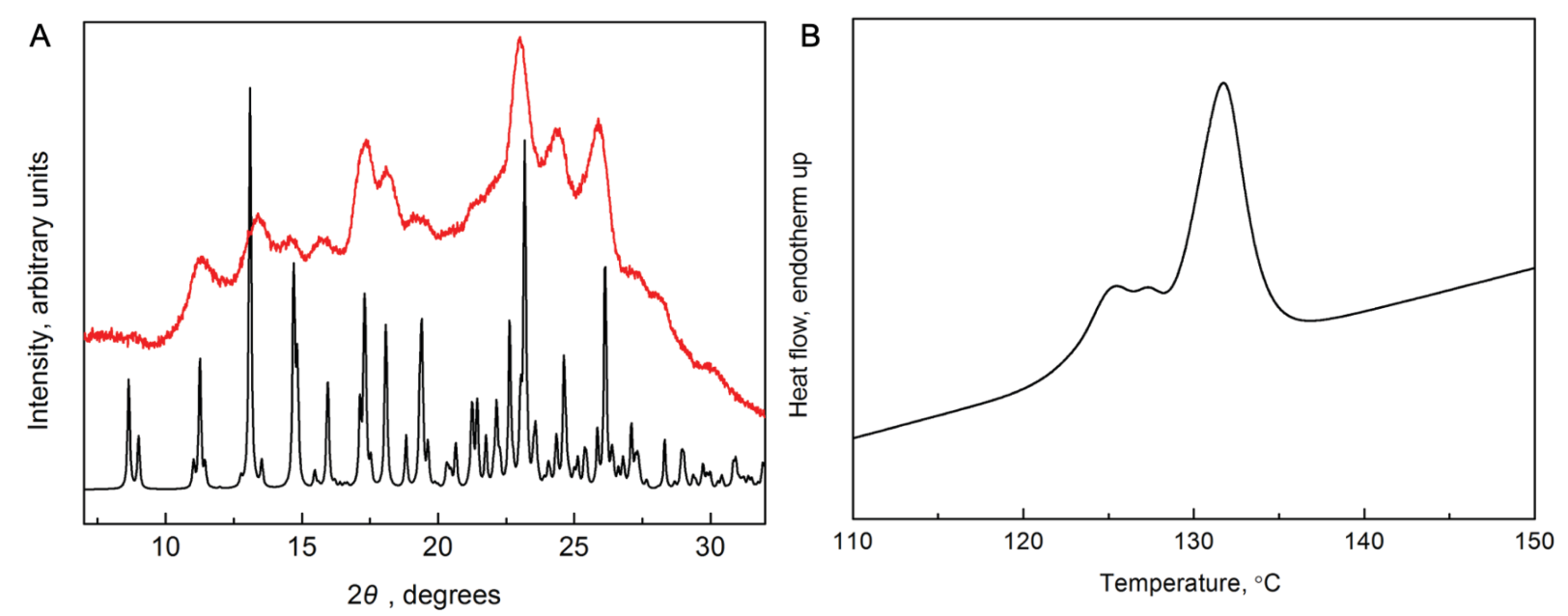

Figure S5. (A) PXRD patterns of FFA mixed with nonporous glass beads. Black line corresponds to the calculated PXRD patterns of Form IV (REFCODE $=$ FPAMCA15). ${ }^{1}$ (B) DSC data for FFA form IV mixed with nonporous glass beads. Sample was heated from 25 to $150{ }^{\circ} \mathrm{C}$ at a rate of $10 \mathrm{~min} /{ }^{\circ} \mathrm{C}$.
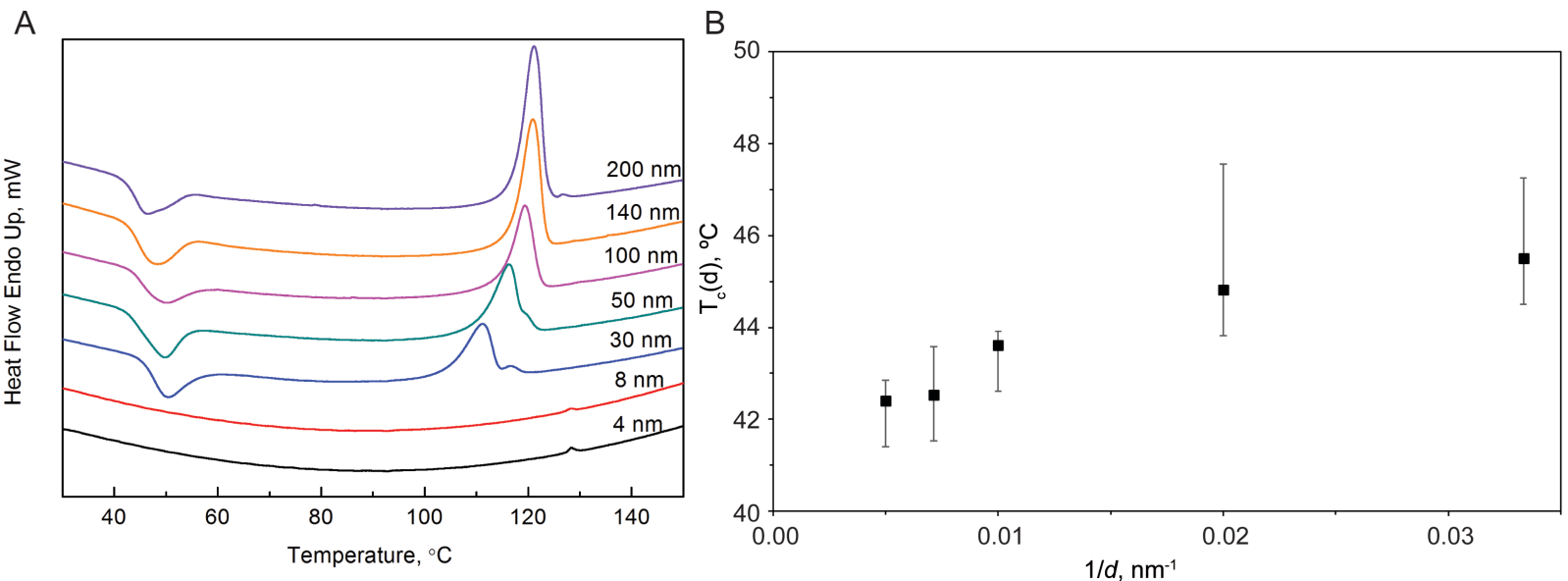

Figure S6. (A) Representative DSC thermographs for FFA form VIII nanocrystals embedded within (a) $30 \mathrm{~nm}$, (b) 50 $\mathrm{nm}$, (c) $100 \mathrm{~nm}$, (d) $140 \mathrm{~nm}$, and (e) $200 \mathrm{~nm}$ CPG pores. The exothermic peaks at ca. $50{ }^{\circ} \mathrm{C}$ were attributed to the crystallization of form VIII from an amorphous phase. The heating and cooling of FFA form VIII was performed at the rate of $10{ }^{\circ} \mathrm{C} / \mathrm{min}$. Additional endotherms occurring at higher melting temperatures could not be assigned. Crystallization and melting peaks were not observed in 4 and $8 \mathrm{~nm}$ pores. (B) Dependence of the crystallization temperature, $T_{\mathrm{c}}$, on the inverse of the pore diameter, $1 / d$, for form VIII. The diameters correspond to the nominal CPG pore sizes, as determined by the vendor. 


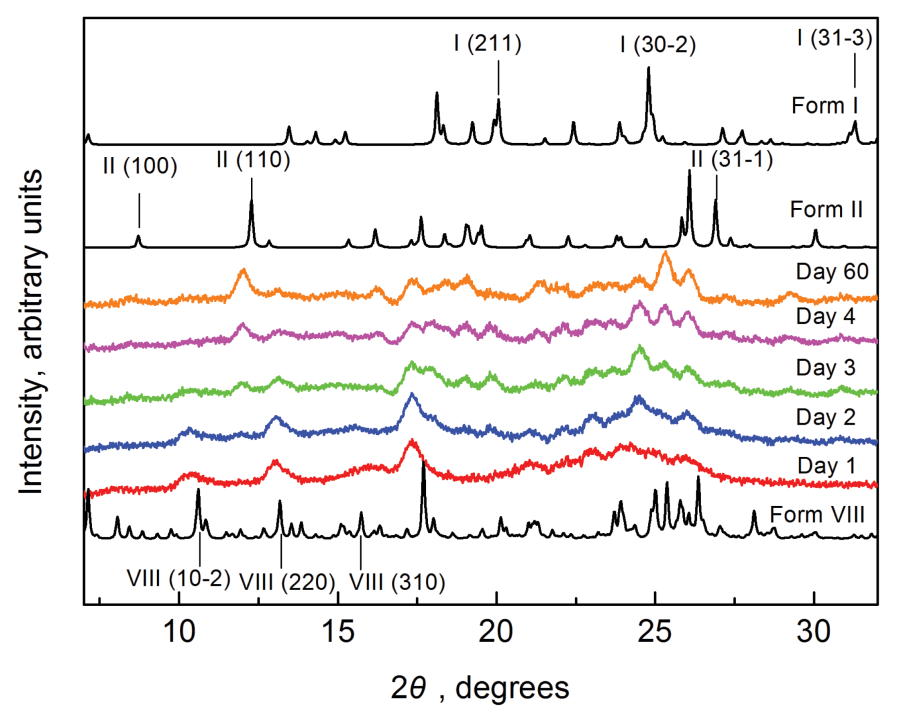

Figure S7. PXRD patterns of FFA embedded in $50 \mathrm{~nm}$ CPG pores measured at room temperature. Three black traces correspond to the simulated PXRD patterns of form VIII (REFCODE = FPAMCA13), form II (REFCODE = FPAMCA17) and form I (REFCODE = FPAMCA18), respectively. ${ }^{1}$

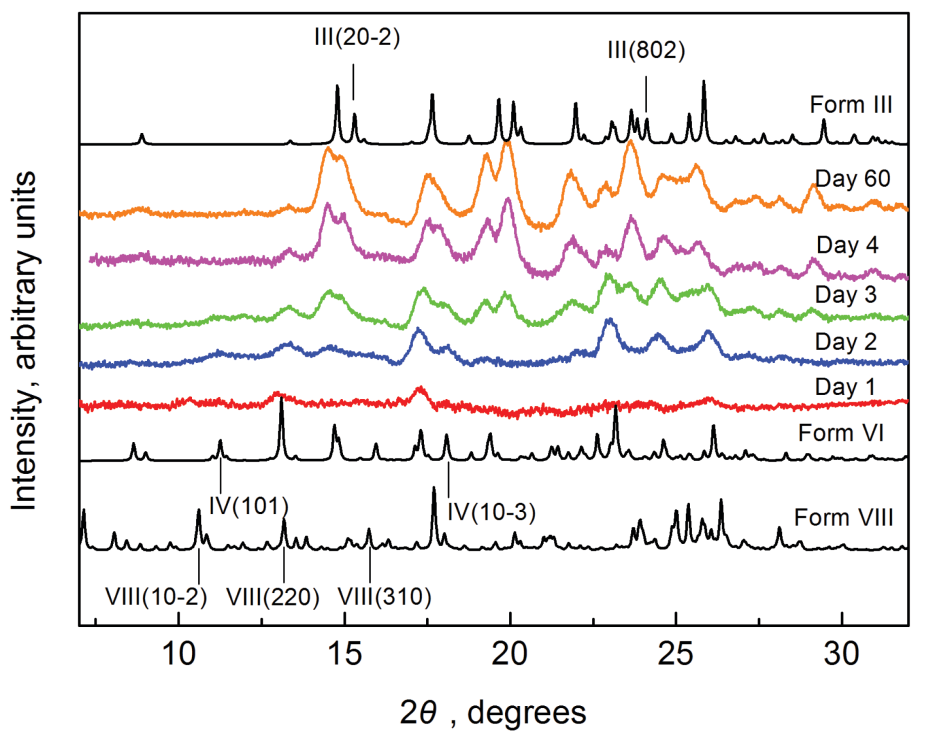

Figure S8. PXRD patterns of FFA embedded in $140 \mathrm{~nm}$ CPG measured at room temperature. Three black traces correspond to simulated PXRD patterns of form VIII (REFCODE = FPAMCA13), form IV (REFCODE = FPAMCA15) and form III (REFCODE = FPAMCA19), respectively. ${ }^{1}$ 


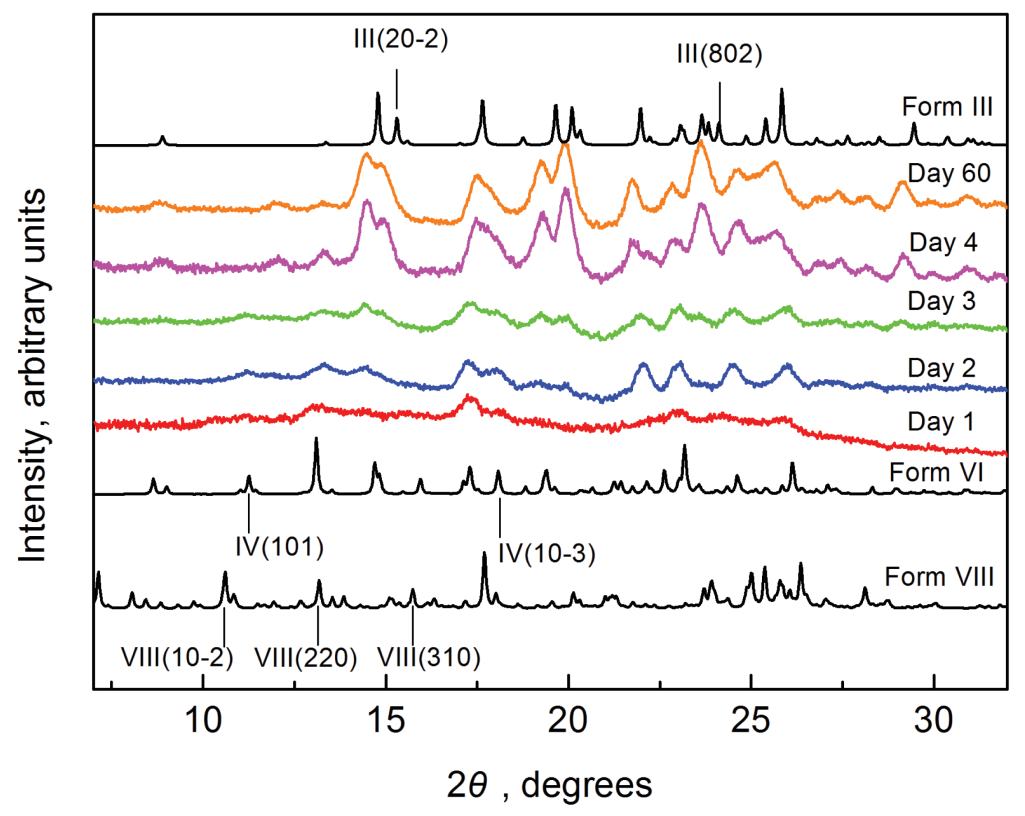

Figure S9. PXRD patterns of FFA embedded in $200 \mathrm{~nm}$ CPG pores measured at room temperature. Three black traces correspond to the simulated PXRD patterns of form VIII (REFCODE = FPAMCA13), form II (REFCODE = FPAMCA17) and form I (REFCODE = FPAMCA18), respectively ${ }^{1}$

\section{REFERENCES}

${ }^{1}$ V. López-Mejías, J. W. Kampf and A. J. Matzger, J. Am. Chem. Soc., 2012, 134, 9872-9875. 